Bull. Korean Math. Soc. 51 (2014), No. 3, pp. 863-881

http://dx.doi.org/10.4134/BKMS.2014.51.3.863

\title{
SOME WARPED PRODUCT SUBMANIFOLDS OF A KENMOTSU MANIFOLD
}

\author{
Viqar Azam Khan and Mohammad Shuaib
}

\begin{abstract}
Many differential geometric properties of a submanifold of a Kaehler manifold are conceived via canonical structure tensors $T$ and $F$ on the submanifold. For instance, a $C R$-submanifold of a Kaehler manifold is a $C R$-product if and only if $T$ is parallel on the submanifold (c.f. $[2])$. Warped product submanifolds are generalized version of $C R$-product submanifolds. Therefore, it is natural to see how the non-triviality of the covariant derivatives of $T$ and $F$ gives rise to warped product submanifolds. In the present article, we have worked out characterizations in terms of $T$ and $F$ under which a contact $C R$ - submanifold of a Kenmotsu manifold reduces to a warped product submanifold.
\end{abstract}

\section{Introduction}

On a submanifold $M$ of an almost Hermitian manifold $(\bar{M}, J, g)$, for any vector field $U$ on $M, J U$ decomposes into tangential and normal parts respectively as $J U=T U+F U$. This defines a one-one tensor field $T$ and a normal valued one-form $F$ on $M$. B. Y. Chen while investigating $C R$-submanifolds of a Kaehler manifold, described many extrinsic geometric properties of the submanifold in terms of the tensor fields $T$ and $F$. For instance, "a $C R$ - submanifold $M$ of a Kaehler manifold is a $C R$-product if and only if $T$ is parallel on $M$ " (cf. [2]). More generally, " $T$ is parallel on a submanifold $M$ of a Kaehler manifold if and only if $M$ is a Riemannian product $N_{1} \times N_{2} \times \cdots \times N_{k}$, where each $N_{i}$ is either a Kaehler submanifold, a totally real submanifold or a Kaehlerian slant submanifold" (cf. [3]). Similar conditions are available in terms of the one-form $F$ as well. Warped product manifolds are not only a generalized version of Riemannian product of two manifolds but also provide an excellent setting to model space-time near black holes and bodies with high gravitational fields (cf. [7]). Thus, as a step forward, V. A. Khan et al. [9] worked out characterizations involving $\bar{\nabla} T$ and $\bar{\nabla} F$ under which a $C R$-submanifold of a Kaehler manifold reduces to a warped product submanifold. On the other hand, I. Hesigawa and I. Mihai [5] as well as by M. I. Munteanu [11] worked

Received May 21, 2013.

2010 Mathematics Subject Classification. 53C25, 53D15.

Key words and phrases. $C R$-submanifold, warped product, Kenmotsu manifold. 
out necessary and sufficient conditions involving the shape operator of the immersion of a contact $C R$-submanifold into a Sasakian manifold under which the submanifold is a warped product submanifold.

We have sought in the present article, characterizations that could turn a contact $C R$-submanifold of a Kenmotsu manifold to a warped product submanifold. This is interesting because Kenmotsu manifolds are themselves warped product manifolds. To this end we realized that if the structure vector field $\xi$ is tangential to a proper contact $C R$-submanifold, the structure tensor $T$ can not be parallel on $M$. This paves way to investigate how the non-triviality of the covariant derivatives of $T$ and $F$ on $M$ yield a warped product submanifold. In fact we have worked out formulas involving $\bar{\nabla} T$ and $\bar{\nabla} F$ which turn a contact $C R$-submanifold of a Kenmotsu manifold, a warped product submanifold. To achieve the object, we have obtained some initial results on the contact $C R$ submanifold of a Kenmotsu manifold in Section 3. These results are used in obtaining the said characterizations in Sections 4 and 5 .

\section{Preliminaries}

Let $\bar{M}$ be a $(2 n+1)$ dimensional almost contact manifold with almost contact structures $(\phi, \xi, \eta)$, where $\phi$ is a $(1,1)$ tensor field, $\xi$ a vector field and $\eta$, a 1 form satisfying

$$
\phi^{2}=-I+\eta \otimes \xi, \eta(\xi)=1, \phi \xi=0 .
$$

On an almost contact manifold there exists a Riemannian metric $g$ which is compatible with the contact structures $(\phi, \xi, \eta)$ in the sense that

$$
g(\phi U, \phi V)=g(U, V)-\eta(U) \eta(V)
$$

for any $U \in T \bar{M}$. One can deduce from the above that

$$
\eta(U)=g(U, \xi) .
$$

In this case the Riemannian manifold $(\bar{M}, g)$ is called an almost contact metric manifold.

If $\bar{\nabla}$ is the Levi-Civita connection on $\bar{M}$, then we have

$$
\left(\bar{\nabla}_{U} \phi\right) V=\bar{\nabla}_{U} \phi V-\phi \bar{\nabla}_{U} V \text {. }
$$

Almost contact metric structures $(\phi, \xi, \eta, g)$ are said to define a Kenmotsu structure on $\bar{M}$ if the following characterizing tensorial equation is satisfied (cf. [8])

$$
\left(\bar{\nabla}_{U} \phi\right) V=g(\phi U, V) \xi-\eta(V) \phi U \text {. }
$$

One can deduce from the above relations that

$$
\bar{\nabla}_{U} \xi=U-\eta(U) \xi
$$

It is also known that Kenmotsu manifolds are warped product manifolds of the type $L \times{ }_{f} F$, where $L$ is a line and $F$ is a Kaehler manifold.

Let $M$ be a submanifold of an almost contact metric manifold $\bar{M}$ with $T M$ and $T^{\perp} M$ as the tangent and normal bundles on $M$, respectively. If $\nabla$ and $\nabla^{\perp}$ 
are the induced Riemannian connections on $T M$ and $T^{\perp} M$, then Gauss and Weingarten formulae are written as:

$$
\begin{aligned}
& \bar{\nabla}_{U} V=\nabla_{U} V+h(U, V), \\
& \bar{\nabla}_{U} N=-A_{N} U+\nabla_{U}^{\perp} N,
\end{aligned}
$$

for any $U, V \in T M$ and $N \in T^{\perp} M . A_{N}$ and $h$, respectively, denote the shape operator (corresponding to the normal vector field $N$ ) and the second fundamental form of the immersion of $M$ into $\bar{M}$. The two are related as

$$
\left.g\left(A_{N} U, V\right)=g(h(U, V)), N\right)
$$

where $g$ denotes the Riemannian metric on $\bar{M}$ as well as the induced metric on $M$.

For any $U \in T M$, we write

$$
T U=\tan (\phi U) \text { and } F U=\operatorname{nor}(\phi U) .
$$

Similarly, for $N \in T^{\perp} M$, we write

$$
t N=\tan (\phi N) \text { and } f N=\operatorname{nor}(\phi N),
$$

where 'tan' and 'nor' are the natural projections associated with the direct decomposition:

$$
T_{x} \bar{M}=T_{x} M \oplus T_{x}^{\perp} M, x \in M .
$$

The tensor fields on $M$ determined by the endomorphism $T$ and the normal valued 1 -form $F$ are denoted by the same letters $T$ and $F$, respectively. Similarly, $t$ and $f$ are tangential and normal valued $(1,1)$-tensor fields on the normal bundle of $M$. The covariant differentiations of the tensor fields $P, F, t$ and $f$ are defined, respectively, as:

$$
\begin{gathered}
\left(\bar{\nabla}_{U} T\right) V=\nabla_{U} T V-T \nabla_{U} V, \\
\left(\bar{\nabla}_{U} F\right) V=\nabla_{U}^{\perp} F V-F \nabla_{U} V, \\
\left(\bar{\nabla}_{U} t\right) N=\nabla_{U} t N-t \nabla_{U}^{\perp} N, \\
\left(\bar{\nabla}_{U} f\right) N=\nabla_{U}^{\perp} f N-f \nabla_{U}^{\perp} N .
\end{gathered}
$$

Let $M$ be a submanifold tangent to the structure vector field $\xi$ isometrically immersed into an almost contact metric manifold $\bar{M}$. Then $M$ is called a contact $C R$-submanifold if it is endowed with the pair of orthogonal distributions $D$ and $D^{\perp}$ satisfying

(i) $T M=D \oplus D^{\perp} \oplus\langle\xi\rangle$, where $\langle\xi\rangle$ is the one dimensional distribution spanned by structure field $\xi$,

(ii) the distribution $D$ is invariant by $\phi$, i.e., $\phi D_{x}=D_{x}$ for each $x \in M$,

(iii) the distribution $D^{\perp}$ is anti-invariant, i.e., $\phi D_{x}^{\perp} \subseteq T_{x}^{\perp} M$ for each $x \in M$.

A contact $C R$-submanifold is proper, if neither $D$ nor $D^{\perp}$ is trivial. A submanifold of an almost contact metric manifold is called a contact $C R$-product if it is locally a Riemannian product of a $\phi$-invariant submanifold $M_{T}$ and a 
$\phi$-anti-invariant submanifold $M_{\perp}$ of $\bar{M}$ (cf. [15]). Hence, every $C R$-product is a $C R$-submanifold but the converse is not true in general. However, if the two distributions on a $C R$-submanifold are parallel then the submanifold is a contact $C R$-product.

The notion of warped product (or more generally warped bundle) was introduced by R. L. Bishop and B. O'Neill [1] in order to construct a large variety of manifolds of negative curvature and are a generalized version of Riemannian product of two manifolds. We recall in the following paragraphs the notion of warped product manifolds.

Let $\left(M_{1}, g_{1}\right)$ and $\left(M_{2}, g_{2}\right)$ be two Riemannian manifolds with Riemannian metrics $g_{1}$ and $g_{2}$ respectively and $f$ be a positive differentiable function on $M_{1}$. Then the warped product $M_{1} \times_{f} M_{2}$ is the manifold $M_{1} \times M_{2}$ endowed with Riemannian metric $g$ given by

$$
g=\pi_{1}^{*}\left(g_{1}\right)+\left(f \circ \pi_{1}\right)^{2} \pi_{2}^{*}\left(g_{2}\right),
$$

where $\pi_{i}(i=1,2)$ are the projection maps of $M$ onto $M_{1}$ and $M_{2}$, respectively. The function $f$ in this case, is known as the warping function (cf. [1]). If the warping function $f$ is just a constant, the warped product is simply a Riemannian product, known as a trivial warped product. More generally, a trivial warped product is a Riemannian product $M_{1} \times M_{2}^{f}$ where $M_{2}^{f}$ is the manifold with Riemannian metric $f^{2} g_{2}$ which is homothetic to the metric $g_{2}$ of $M_{2}$.

A warped product manifold isometrically immersed into a Riemannian manifold is known as warped product submanifold.

Bishop and O'Neill obtained many interesting geometric properties of a warped product manifold. We enlist in the following some of the observations and formulas relevant to our study.

Theorem 2.1 ([1]). Let $M=M_{1} \times{ }_{f} M_{2}$ be a warped product manifold. If $X, Y \in T M_{1}$ and $Z, W \in T M_{2}$, then

(i) $\nabla_{X} Y \in T M_{1}$,

(ii) $\nabla_{X} Z=\nabla_{Z} X=(X f / f) Z$,

(iii) $\operatorname{nor}\left(\nabla_{Z} W\right)=-(g(Z, W) / f) \nabla f$,

where $\operatorname{nor}\left(\nabla_{Z} W\right)$ denotes the component of $\nabla_{Z} W$ in $T M_{1}$ and $\nabla f$ is the gradient of $f$ defined as

$$
g(\nabla f, U)=U f
$$

for any $U \in T M$.

A couple of important consequences of the above theorem can be stated as:

Corollary 2.2. On a warped product manifold $M=M_{1} \times_{f} M_{2}$,

(i) $M_{1}$ is totally geodesic in $M$.

(ii) $M_{2}$ is totally umbilical in $M$. 


\section{Contact $C R$-submanifolds of a Kenmotsu manifold}

Throughout, we assume that $\bar{M}$ is a Kenmotsu manifold and $M$, a contact $C R$-submanifold of $\bar{M}$. Thus, the tangent bundle $T M$ and the normal bundle $T^{\perp}(M)$ of $M$ admit the following direct decompositions

$$
T M=D \oplus D^{\perp} \oplus\langle\xi\rangle,
$$

and,

$$
T^{\perp}(M)=\phi D^{\perp} \oplus \nu
$$

where $\nu$ is the orthogonal complement of $\phi D^{\perp}$ in $T^{\perp}(M)$ and is invariant under $\phi$. It is also easy to observe that for any $N \in T^{\perp} M, t N \in D^{\perp}$ and $f N \in \nu$. Further, for any $U \in T M, T U \in D$ and $F U \in \phi D^{\perp}$.

Now, in view of the decomposition of the tangent bundle, for any $U \in T M$, we may write

$$
U=B U+C U+\eta(U) \xi
$$

where $B U \in D$ and $C U \in D^{\perp}$. Following are some easy observations:

$$
\begin{gathered}
T^{2} U=-U+\eta(U) \xi, \\
T B U=T U, F C U=F U, \\
t F U=-C U, F B U=0, T C U=0 .
\end{gathered}
$$

As $\xi$ is assumed to be tangential to the submanifold, by (5) and (6),

$$
\text { (a) } \quad \nabla_{U} \xi=U-\eta(U) \xi, \quad \text { (b) } \quad h(U, \xi)=0
$$

for each $U \in T M$. Further, as an immediate consequence of $(21)(\mathrm{b})$, we have

$$
A_{F U} \xi=0 \text {. }
$$

Since our aim is to study contact $C R$-submanifolds as warped product submanifolds, we need to ensure the existence of $\phi$-invariant and $\phi$-anti-invariant factors of the submanifolds. To this end, we have:

Lemma 3.1. Let $M$ be a contact $C R$-submanifold of a Kenmotsu manifold $\bar{M}$. Then

for each $Z, W \in D^{\perp}$.

$$
A_{\phi W} Z=A_{\phi Z} W
$$

Proof. For each $U \in T M$ and $Z \in D^{\perp}$, it follows from (4) that $\left(\bar{\nabla}_{U} \phi\right) Z=0$, Therefore, by (3), $\bar{\nabla}_{U} \phi Z=\phi \bar{\nabla}_{U} Z$, which on using Gauss and Weingarten formulae yields

$$
g\left(A_{\phi W} Z, U\right)=g\left(A_{\phi Z} W, U\right)
$$

for each $U \in T M$ and $Z, W \in D^{\perp}$. This proves the assertion.

Theorem 3.2. The $\phi$-anti-invariant distributions $D^{\perp}$ and $D^{\perp} \oplus\langle\xi\rangle$ on a contact CR-submanifold of a Kenmotsu manifold are involutive. 
Proof. For $Z, W \in D^{\perp}$ and $U \in T M$,

$$
g(T[Z, W], U)=g(\phi[Z, W], U)=g\left(\phi \bar{\nabla}_{Z} W-\phi \bar{\nabla}_{W} Z, U\right) .
$$

On using (3) and (4), the above equation takes the form

$$
g(T[Z, W], U)=g\left(\bar{\nabla}_{Z} \phi W-\bar{\nabla}_{W} \phi Z, U\right),
$$

which on applying Weingarten formula reduces to

$$
g(T[Z, W], U)=g\left(A_{\phi Z} W-A_{\phi W} Z, U\right) .
$$

The right hand side of the above vanishes by virtue of Lemma (3.1) implying that $[Z, W] \in D^{\perp}$. This proves that $D^{\perp}$ is involutive. Further, as $[Z, \xi] \in$ $D^{\perp} \oplus\langle\xi\rangle$ for any $Z \in D^{\perp}, D^{\perp} \oplus\langle\xi\rangle$ is involutive.

Now, on using (4) and (6), it is easy to prove that

$$
g\left(A_{\phi Z} W, X\right)=g\left(\nabla_{W} Z, \phi X\right)
$$

for each $X \in D \oplus\langle\xi\rangle$ and $Z, W \in D^{\perp}$

From the above observation we may deduce that:

Theorem 3.3. A necessary and sufficient condition for a $\phi$-anti-invariant submanifold $M_{\perp}$ to be totally geodesic in $M$ is that

$$
g\left(h(X, Z), \phi D^{\perp}\right)=0
$$

for each $X \in D \oplus\langle\xi\rangle$ and $Z \in D^{\perp}$.

With regard to the $\phi$-invariant distribution, it is known that:

Theorem 3.4 ([10]). Let $M$ be a contact CR-submanifold of a Kenmotsu manifold. Then the $\phi$-invariant distributions $D$ and $D \oplus\{\xi\}$ are involutive if and only if

$$
g(h(T X, Y), F Z)=g(h(X, T Y), F Z)
$$

for $X, Y \in D$ and $Z \in D^{\perp}$.

B. Y. Chen [2], proved that a $C R$-submanifold $M$ of a Kaehler manifold $\bar{M}$ is a $C R$-product if and only if the structure tensor $T$ is parallel on $M$. Extending the result to the contact setting, M. I. Munteanu [11] obtained the following:

Theorem 3.5 ([11]). Let $M$ be a contact $C R$-submanifold of a Sasakian manifold, with $\xi \in D$. Then $M$ is a contact $C R$-product if and only if $T$ satisfies:

$$
\left(\bar{\nabla}_{U} T\right) V=-g(B U, V) \xi+\eta(V) B U
$$

for all $U, V \in T M$.

Now, for a submanifold $M$ of a Kenmotsu manifold $\bar{M}$, on making use of equations (3)-(13), we obtain

$$
\begin{gathered}
\left(\bar{\nabla}_{U} T\right) V=A_{F V} U+t h(U, V)-\eta(V) T U-g(U, T V) \xi \\
\left(\bar{\nabla}_{U} F\right) V=f h(U, V)-h(U, T V)-\eta(V) F U .
\end{gathered}
$$

It is easy to deduce from the above formulas that: 
Lemma 3.6. On a contact $C R$-submanifold $M$ of a Kenmotsu manifold $\bar{M}$,

$$
\begin{array}{llll}
\text { (a) } & \left(\bar{\nabla}_{\xi} T\right) U=0, & \text { (b) } & \left(\bar{\nabla}_{U} T\right) \xi=-T U, \\
\text { (c) } & \left(\bar{\nabla}_{\xi} F\right) U=0, & \text { (d) } & \left(\bar{\nabla}_{U} F\right) \xi=-F U
\end{array}
$$

for each $U \in T M$.

Theorem 3.7. On a contact proper CR-submanifold of a Kenmotsu manifold $\bar{M}, T$ can not be parallel.

Proof. Suppose $\bar{\nabla} T=0$ on a contact $C R$-submanifold $M$ of a Kenmotsu manifold then by formula (25),

$$
t h(X, Y)=g(X, T Y) \xi+\eta(Y) T X
$$

for each $X, Y \in D \oplus\langle\xi\rangle$. Since $t h(X, Y) \in D^{\perp}$, it follows from the above equation and the orthogonality of the distributions in $T M$, that $\operatorname{th}(X, Y)=0$, and

$$
g(X, T Y) \xi=-\eta(Y) T X
$$

which means $D=\langle\xi\rangle$ and is therefore parallel. That shows, each leaf $M_{T}$ of $D$ (the integral curve of $\xi$ ) is totally geodesic in $M$. Moreover, as equation (23) is automatically satisfied in this case, each leaf $M_{\perp}$ of $D^{\perp}$ is also totally geodesic in $M$. Hence, under the parallelism of $T, M$ is a contact $C R$-product between an integral curve of $\xi$ and a $\phi$-anti-invariant submanifold $M_{\perp}$ of $\bar{M}$. This proves the Theorem.

\section{Contact $C R$-warped product submanifolds of a Kenmotsu manifold}

B. Y. Chen [4] initiated the study of warped product manifolds with extrinsic geometric point of view by exploring $C R$-submanifolds of Kaehler manifolds as warped products.

Let $M_{1}$ and $M_{2}$ be submanifolds of a Kenmotsu manifold $\bar{M}$ such that a warped product manifold $M=M_{1} \times_{f} M_{2}$ admit an isometric immersion into $\bar{M}$ with $\xi$ tangential to $M$. Then for any $U_{1}, V_{1} \in T M_{1}, U_{2}, V_{2} \in T M_{2}$, by part (ii) of Theorem 2.1,

$$
\nabla_{U_{1}} U_{2}=\left(U_{1} \ln f\right) U_{2}
$$

whereas by part (iii) of Theorem 2.1, we have

$$
\operatorname{nor}\left(\nabla_{U_{2}} V_{2}\right)=-g\left(U_{2}, V_{2}\right) \nabla \ln f
$$

If $\xi_{1}$ and $\xi_{2}$ are the components of $\xi$ along $M_{1}$ and $M_{2}$, respectively, then

$$
\nabla_{U} \xi=\nabla_{U} \xi_{1}+\nabla_{U} \xi_{2}
$$

for any $U \in T M$. Now, by $(21)(\mathrm{a}), \nabla_{U_{1}} \xi=U_{1}-\eta\left(U_{1}\right) \xi$ which on making use of (29) and (31) gives

$$
\nabla_{U_{1}} \xi_{1}+\left(U_{1} \ln f\right) \xi_{2}=U_{1}-\eta\left(U_{1}\right) \xi_{1}-\eta\left(U_{1}\right) \xi_{2} .
$$


On comparing components tangential to $M_{1}$ in (32) while taking account of the fact that $\nabla_{U_{1}} \xi_{1} \in T M_{1}$, we get

$$
\nabla_{U_{1}} \xi_{1}=U_{1}-\eta\left(U_{1}\right) \xi_{1}
$$

whereas on equating components tangential to $M_{2}$, we get

$$
\left(U_{1} \ln f\right) \xi_{2}=-\eta\left(U_{1}\right) \xi_{2} .
$$

If $\xi_{2} \neq 0$, then on using (2) and (16), the above equation yields

$$
\nabla \ln f=-\xi_{1} .
$$

Similarly, for $U_{2} \in T M_{2}$, applying (29), (30), (31) on the equation $\nabla_{U_{2}} \xi=$ $U_{2}-\eta\left(U_{2}\right) \xi$ and comparing components tangential to $M_{1}$ and $M_{2}$, we obtain

$$
\eta\left(U_{2}\right) \nabla \ln f=\eta\left(U_{2}\right) \xi_{1},
$$

and,

$$
\tan \left(\nabla_{U_{2}} \xi_{2}\right)=\left(1-\xi_{1} \ln f\right) U_{2}-\eta\left(U_{2}\right) \xi_{2},
$$

where tan denotes the component of the underlying vector field along $M_{2}$.

If $\xi_{2}=0$, then from (36), we have,

$$
(\xi \ln f) U_{2}=U_{2} .
$$

That means, in this case if $M_{2}$ is non trivial, then $\nabla \ln f=\xi$, that is $f(t)=e^{t}$, where $\xi=\frac{\partial}{\partial t}$.

On the other hand if $\xi_{2} \neq 0$, then by (35), we have

$$
\xi_{1}=\nabla \ln f .
$$

Hence, in case of $\xi_{2} \neq 0$, it follows from (34) and (37) that

$$
\nabla \ln f=0 \text {. }
$$

That shows that if $\xi$ has non-zero component along $M_{2}$, then the warped product $M$ is trivial, that is, in this case $M$ is simply a Riemannian product of $M_{1}$ and $M_{2}$. To summarize the above observations, we state:

Theorem 4.1. Let $M=M_{1} \times_{f} M_{2}$ be a warped product submanifold of a Kenmotsu manifold $\bar{M}$ such that the structure vector field $\xi$ is tangential to the submanifold $M$. If $\xi$ has non-trivial component along the second factor $M_{2}$ of $M$, then $M$ is a Riemannian product of $M_{1}$ and $M_{2}$ (i.e., a trivial warped product). That means there does not exist a non-trivial warped product submanifold in a Kenmotsu manifold such that $\xi$ is tangential to the second factor of $M$. However, non-trivial warped product submanifolds $M_{1} \times{ }_{f} M_{2}$ do exist in a Kenmotsu manifold if $\xi$ is tangential to the first factor of $M$. In this case, the warping function $f$ is given by $f(t)=e^{t}$, where $\xi=\frac{\partial}{\partial t}$.

Since $T$ can not be parallel on a contact $C R$-submanifold $M$ of a Kenmotsu manifold $\bar{M}$ (cf. Theorem 3.7), one can look for characterizations in term of $\bar{\nabla} T$ and $\bar{\nabla} F$ under which a contact $C R$-submanifold of $\bar{M}$ is a $C R$-warped product and in particular a $C R$-product. This is a relevant problem in view 
of the fact that along the years there has been interests to find an analogous of classical de Rham theorem to warped products. The following theorem was proved by Hiepko [6] that we will be using to give a characterization of warped product submanifolds.

Theorem 4.2. Let $F$ be a vector sub bundle in the tangent bundle of a Riemannian manifold $M$ and let $F^{\perp}$ be its normal bundle. Assume that the two distributions are both involutive and the integral manifold of $F\left(\right.$ resp. $\left.F^{\perp}\right)$ are extrinsic spheres (resp. totally geodesic). Then $M$ is locally isometric to a warped product $M_{1} \times_{f} M_{2}$. Moreover, if $M$ is simply connected and complete there exists a global isometry of $M$ with a warped product.

If the two factors of a warped product submanifold are $\phi$-invariant and $\phi$ anti-invariant submanifolds, then there are two possible warped product submanifolds namely (i) $M_{T} \times_{f} M_{\perp}$ and (ii) $M_{\perp} \times_{f} M_{T}$, where $M_{T}$ and $M_{\perp}$ are $\phi$-invariant and $\phi$-anti-invariant submanifolds respectively of a Kenmotsu manifold $\bar{M}$. The structure vector field $\xi$ in the two cases remains tangential to $M_{T}$ and $M_{\perp}$, respectively. The warped product submanifolds of type (i) are called as $C R$-warped product submanifold whereas warped product submanifolds of type (ii) are known as warped product $C R$-submanifolds. These warped product submanifolds reduce to $C R$-products if the warping function $f$ is a constant function.

Throughout, we will be denoting the by $X, Y, \ldots$, etc., the vector fields belonging to $\phi$-invariant distribution and by $Z, W, \ldots$, etc., the vector fields belonging to the $\phi$-anti-invariant distribution. With these convention, on a $C R$-warped product submanifold of a Kenmotsu manifold, by (29), we have

$$
\nabla_{X} Z=\nabla_{Z} X=(X \ln f) Z \text {. }
$$

As an immediate consequence of (38), (11) and the fact that $T Z=0$, we may obtain

$$
\left(\bar{\nabla}_{X} T\right) Z=0
$$

and,

$$
\left(\bar{\nabla}_{Z} T\right) X=(T X \ln f) Z .
$$

More generally, we prove:

Lemma 4.3. Let $M$ be a contact $C R$-warped product submanifold of a Kenmotsu manifold. Then

(i) $\left(\bar{\nabla}_{U} T\right) X=(T X \ln f) C U-\eta(X) T U+g(T U, X) \xi$,

(ii) $\left(\bar{\nabla}_{U} T\right) Z=g(C U, Z) T \nabla \ln f$

(iii) $\left(\bar{\nabla}_{U} F\right) X=-(X \ln f) F C U$,

(iv) $\left(\bar{\nabla}_{U} F\right) Z=f h(U, Z)$ for each $U \in T M, X \in T M_{T}$ and $Z \in T M_{\perp}$.

Proof. By formula (11) and the fact that $M_{T}$ is totally geodesic in $M$, it follows that $\left(\bar{\nabla}_{Y} T\right) X$ lies on $M_{T}$, whereas $t h(X, Y)$ lies on $M_{\perp}$. Taking account of 
these observations while comparing the components tangential to $M_{\perp}$ and $M_{T}$ respectively in formula $(25)$, we find that $h(X, Y) \in \nu$ and

$$
\left(\bar{\nabla}_{Y} T\right) X=g(X, T Y) \xi-\eta(X) T Y .
$$

Further, on using (40) and (27)(a) we get

$$
\left(\bar{\nabla}_{Z} T\right) X=(T X \ln f) Z
$$

and,

$$
\left(\bar{\nabla}_{\xi} T\right) X=0 .
$$

Combining the above observations while making use of (17), we get:

$$
\left(\bar{\nabla}_{U} T\right) X=-\eta(X) T B U+(T X \ln f) C U+g(T B U, X) \xi .
$$

This proves statement (i).

Now, by (17), we may write

$$
\left(\bar{\nabla}_{U} T\right) Z=\left(\bar{\nabla}_{B U} T\right) Z+\left(\bar{\nabla}_{C U} T\right) Z+\eta(U)\left(\bar{\nabla}_{\xi} T\right) Z .
$$

The first and the last term of the above equation are zero by (39) and (27)(a) respectively whereas by $(11)$ and $(30),\left(\bar{\nabla}_{C U} T\right) Z=g(C U, Z) T \nabla \ln f$. Taking account of these observations in the above equation, we get:

$$
\left(\bar{\nabla}_{U} T\right) Z=g(C U, Z) T \nabla \ln f .
$$

This proves statement (ii) of the lemma.

Now, by (17), we may write

$$
\left(\bar{\nabla}_{U} F\right) X=\left(\bar{\nabla}_{B U} F\right) X+\left(\bar{\nabla}_{C U} F\right) X+\eta(U)\left(\bar{\nabla}_{\xi} F\right) X .
$$

The first term in the right hand side of the above equation is zero by virtue of (12) and the fact that $M_{T}$ is totally geodesic on $M$ whereas the last term vanishes by virtue of Lemma (3.6)(c). Thus by applying (12) on the middle term, the equation takes the form:

$$
\left(\bar{\nabla}_{U} F\right) X=-F \nabla_{C U} X
$$

which on making use of (29) establishes part (iii) of the lemma.

Since, $T Z=0=\eta(Z)$, equation(27) yields part(iv) of the lemma.

Now, we may prove:

Theorem 4.4. A contact CR-submanifold $M$ of a Kenmotsu manifold $\bar{M}$, is locally isometric to a $C R$-warped product if and only if

$$
\left(\bar{\nabla}_{U} T\right) V=g(T U, V) \xi+T V(\mu) C U+g(C U, C V) T(\nabla \mu)-\eta(V) T U
$$

for any $U, V \in T M$ where $\mu$ is a $\mathbb{C}^{\infty}$-function on $M$ such that $Z \mu=0$ for all $Z \in D^{\perp}$. 
Proof. Let $M$ be a contact $C R$-warped product submanifold of $\bar{M}$. Then by (17) and $(27)(\mathrm{b})$, we may write

$$
\left(\bar{\nabla}_{U} T\right) V=\left(\bar{\nabla}_{U} T\right) B V+\left(\bar{\nabla}_{U} T\right) C V-\eta(V) T U
$$

On applying Lemma 4.3 the above equation takes the form

$$
\left(\bar{\nabla}_{U} T\right) V=g(T U, B V) \xi+T V(\ln f) C U+g(C U, C V) T(\nabla \ln f)-\eta(V) T U .
$$

As $T U \in D$, the first term in the right hand side of the above equation is same as $g(T U, V)$. This verifies (42).

Conversely suppose that $M$ is contact $C R$-submanifold of $\bar{M}$ such that equation (42) is satisfied on $M$ with $\mu$ a $C^{\infty}$ - function on $M$. Then choosing $X, Y \in D \oplus\langle\xi\rangle$, and taking account of the fact that $C X=C Y=0$, equation (42) yields that

$$
g\left(\left(\bar{\nabla}_{X} T\right) Y, Z\right)=g\left(\nabla_{X} T Y, Z\right)=0
$$

for each $Z \in D^{\perp}$. This proves that $D \oplus\langle\xi\rangle$ is parallel. In other words $D \oplus\langle\xi\rangle$ is integrable and each of its leaves $M_{T}$ is totally geodesic in $M$. It is also straightforward to see from equation (42) that

$$
\left(\bar{\nabla}_{Z} T\right) X=(T X \mu) Z \text {. }
$$

Taking product with $W \in D^{\perp}$ in both sides of the above equation while using (11) and (16), we get

$$
g\left(\nabla_{Z} W, T X\right)=-g(T X, \nabla \mu) g(Z, W) .
$$

Since, $D^{\perp}$ is involutive by Theorem 3.2, denoting the second fundamental form of the immersion of $M_{\perp}$ (a leaf of $D^{\perp}$ ) into $M$ by $h^{\perp}$ and using the Gauss formula on the above equation, we get

$$
g\left(\left(h^{\perp}(Z, W), T X\right)=-g(T X, \nabla \mu) g(Z, W) .\right.
$$

This implies that

$$
h^{\perp}(Z, W)=-g(Z, W) \nabla \mu .
$$

That means $M_{\perp}$ is totally umbilical in $M$ with $\nabla \mu$ as the mean curvature with respect to the immersion of $M_{\perp}$ into $M$. Further, $\nabla \mu$ is parallel as $Z \mu=o$. That is, the leaves of $D^{\perp}$ are extrinsic spheres in $M$. Hence, by virtue of Theorem 4.2, $M$ is locally isometric to a $C R$-warped product submanifold $M_{T} \times_{e^{\mu}} M_{\perp}$.

As an immediate consequence of the above theorem, we deduce the following characterization for a contact $C R$-submanifold to be a contact $C R$-product.

Corollary 4.5. Let $M$ be a contact $C R$-submanifold of a Kenmotsu manifold with $\xi \in D$. Then $M$ is a contact $C R$-product if and only if

$$
\left(\bar{\nabla}_{U} T\right) V=g(T U, V) \xi-\eta(V) T U .
$$


The above result can be treated as an analogue of Theorem 3.5 in the setting of contact $C R$ - submanifold of a Kenmotsu manifold.

Now, by (25), we have

$$
A_{\phi Z} X=\left(\bar{\nabla}_{X} T\right) Z-t h(X, Z)
$$

for any $X \in D \oplus\langle\xi\rangle$, and $Z \in D^{\perp}$. Moreover, we also observe from (25) that

$$
\operatorname{th}(X, Z)=\left(\bar{\nabla}_{Z} T\right) X .
$$

Substituting the value of $t h(X, Y)$ from (45) into (44), we get

$$
A_{\phi Z} X=\left(\bar{\nabla}_{X} T\right) Z-\left(\bar{\nabla}_{Z} T\right) X .
$$

By applying (42) in the last equation, we obtain

$$
A_{\phi Z} X=-(\phi X \ln f) Z \text {. }
$$

Now, as the condition (42) is necessary and sufficient, we conclude:

Corollary 4.6. Let $M$ be a contact $C R$-submanifold of a Kenmotsu manifold $\bar{M}$ with non-trivial distributions $D$ and $D^{\perp}$. Then $M$ is locally a contact $C R$ warped product submanifold if and only if

$$
A_{\phi Z} X=-(\phi X \mu) Z, X \in D \oplus\langle\xi\rangle \text { and } Z \in D^{\perp} .
$$

In particular, we have:

Corollary 4.7. A contact $C R$-submanifold of a Kenmotsu manifold is a contact $C R$-product if and only if

$$
A_{\phi Z} X=0
$$

for each $X \in D \oplus\langle\xi\rangle$ and $Z \in D^{\perp}$.

The above result can be treated as an extension of the characterization obtained by B. Y. Chen [2] in the setting of $C R$-submanifolds of a Kaehler manifold, whereas (46) is an extension of Chen's characterization for a $C R$ submanifold of a Kaehler manifold to be $C R$-warped product submanifold (cf. [4]).

In terms of the canonical structure $F$, we have:

Theorem 4.8. A contact CR-submanifold of a Kenmotsu manifold $\bar{M}$ is locally isometric to a $C R$-warped product if and only if

$$
\left(\bar{\nabla}_{U} F\right) V=f h(U, C V)-(B V(\mu)+\eta(V)) F U
$$

for any $U, V \in T M$ where $\mu$ is a $C^{\infty}$-function on $M$ such that $Z \mu=0$ for each $Z \in D^{\perp}$.

Proof. On using (17) and (28), $\left(\bar{\nabla}_{U} F\right) V$ is expressed as

$$
\left(\bar{\nabla}_{U} F\right) V=\left(\bar{\nabla}_{U} F\right) B V+\left(\bar{\nabla}_{U} F\right) C V+\eta(V)\left(\bar{\nabla}_{U} F\right) \xi .
$$


If $M=M_{T} \times_{f} M_{\perp}$ is a contact $C R$-warped product submanifold of $\bar{M}$, then applying on Lemmas 4.3 and 3.6, the last equation reduces to

$$
\left(\bar{\nabla}_{U} F\right) V=-(B V \ln f) F C U+f h(U, C V)-\eta(V) F U .
$$

Now, as $F C U=F U$, we obtain

$$
\left(\bar{\nabla}_{U} F\right) V=f h(U, C V)-((B V \ln f)+\eta(V)) F U .
$$

This verifies (47).

Conversely suppose that (47) holds on a contact $C R$-submanifold of a Kenmotsu manifold $\bar{M}$. Then for $X, Y \in D \oplus\langle\xi\rangle$ formula (47) gives

$$
\left(\bar{\nabla}_{X} F\right) Y=0
$$

which, on applying (12) implies that $\nabla_{X} Y \in D \oplus\langle\xi\rangle$. This shows that $D \oplus\langle\xi\rangle$ is involutive and its leaves are totally geodesic in $M$. Again for $Z \in D^{\perp}$ and $X \in D \oplus\langle\xi\rangle$, by $(47)$

$$
\left(\bar{\nabla}_{Z} F\right) X=-B X(\mu) F Z-\eta(X) F Z .
$$

Taking product with $\phi W$ in both side of the above equation, we get

$$
g\left(\nabla_{Z} W, X\right)=-(B X(\mu)+\eta(X)) g(Z, W)
$$

or,

$$
\begin{aligned}
g\left(\nabla_{Z} W, X\right) & =-[g(\nabla \mu, B X)+\eta(X)] g(Z, W) \\
& =-g(X, \xi-\eta(\nabla \mu) \xi+\nabla \mu) g(Z, W) \\
& =-g(X, B \nabla \mu+\xi) g(Z, W) .
\end{aligned}
$$

Since $\mu$ is a function on $M$ with $Z \mu=0$ for each $Z \in D^{\perp}$, we have $C(\nabla \mu)=0$. Now, it follows from the above that

$$
\operatorname{nor}\left(\nabla_{Z} W\right)=-g(Z, W) \nabla \mu .
$$

If $h^{\perp}$ be the second fundamental form of $M_{\perp}$ in $M$, then it follows from the above that

$$
h^{\perp}(Z, W)=-g(Z, W) \nabla \mu .
$$

This shows that $M_{\perp}$ is totally umbilical in $M$ with $\nabla \mu$ as the mean curvature vector with respect to the immersion of $M_{\perp}$ into $M$. Further $\nabla \mu$ is parallel as $Z \mu=0, \forall Z \in D^{\perp}$. That is, the leaves of $D^{\perp}$ are extrinsic spheres in $M$. Hence, $M$ is locally isometric to a $C R$-warped product by virtue of Theorem 4.2 .

Example 4.9. Let $\mathbb{R}^{2 n+1}=\mathbb{C}^{n} \times \mathbb{R}$ be the $(2 n+1)$-dimensional Euclidean space endowed with the almost contact metric structure $(\phi, \xi, \eta, g)$ defined by

$$
\begin{gathered}
\phi\left(x^{1}, x^{2}, \ldots, x^{2 n}, t\right)=\left(-x^{n+1},-x^{n+2}, \ldots,-x^{2 n}, x^{1}, \ldots, x^{n}, 0\right), \\
\xi=e^{t} \frac{\partial}{\partial t}, \eta=e^{t} d t, \text { and } g=e^{2 t}\langle,\rangle
\end{gathered}
$$


where $\left(x^{1}, x^{2}, \ldots, x^{2 n}, t\right)$ are Cartesian co-ordinates and $\langle$,$\rangle is the Euclidean$ metric on $\mathbb{R}^{2 n+1}$. Then $(\phi, \xi, \eta, g)$ is a trans-Sasakian structure on $\mathbb{R}^{2 n+1}$ (cf. $[13])$.

Consider the immersion $x$ as

$$
x\left(u^{1}, u^{2}, u^{3}, u^{4}, t\right)=\left(u^{1}, 0, u^{3}, 0, u^{2}, 0,0, u^{4}, t\right)
$$

of a 5 -dimensional manifold $M$ into $\left(R^{9}, \phi, \xi, \eta, g\right)$ and choose a frame $\left\{e_{1}, e_{2}\right.$, $\left.e_{3}, e_{4}, e_{5}\right\}$ of orthogonal vector fields on $\bar{M}$ as

$$
\begin{gathered}
e_{1}=\frac{\partial}{\partial x^{1}}+x^{5} \frac{\partial}{\partial t}, e_{2}=\frac{\partial}{\partial x^{5}}, e_{3}=\frac{\partial}{\partial x^{3}}+x^{7} \frac{\partial}{\partial t}, \\
e_{4}=\frac{\partial}{\partial x^{8}}, e_{5}=\frac{\partial}{\partial t}=\xi .
\end{gathered}
$$

If we define the distributions $D=\operatorname{span}\left\{e_{1}, e_{2}, e_{5}\right\}$ and $D^{\perp}=\operatorname{span}\left\{e_{3}, e_{4}\right\}$, then $D$ and $D^{\perp}$ are, respectively, $\phi$-invariant and $\phi$-anti-invariant distributions on $M$, giving a $C R$-warped product submanifold $M_{T} \times_{e^{t}} M_{\perp}$ of $\bar{M}$, where $M_{T}$ and $M_{\perp}$ denote the leaves of $D$ and $D^{\perp}$, respectively.

\section{Warped product contact $C R$-submanifolds of a Kenmotsu manifold}

Throughout this section, we assume that $M=M_{\perp} \times_{f} M_{T}$ is a warped product contact proper $C R$-submanifold isometrically immersed into a Kenmotsu manifold $\bar{M}$ with structure vector field tangential to $M_{\perp}$, where $M_{\perp}$ and $M_{T}$ are respectively $\phi$-anti-invariant and $\phi$-invariant submanifolds of $\bar{M}$. In this setting, formula (29) can be written as:

$$
\nabla_{X} Z=\nabla_{Z} X=(Z \ln f) X
$$

for each $X \in T M_{T}$ and $Z \in T M_{\perp}$. As an immediate consequence of (49) and (11), we have

$$
\left(\bar{\nabla}_{Z} T\right) X=0
$$

$$
\left(\bar{\nabla}_{X} T\right) Z=-(Z \ln f) T X .
$$

As $M_{\perp}$ is totally geodesic in $M, T \nabla_{Z} W=0$ for each $Z, W \in D^{\perp}$. Therefore by (11),

$$
\left(\bar{\nabla}_{Z} T\right) W=0 .
$$

Now, for $Z=\xi$, equation (51) becomes

$$
\left(\bar{\nabla}_{X} T\right) \xi=-(\xi \ln f) T X .
$$

On the other hand, by (25),

$$
\left(\nabla_{X} T\right) \xi=-T X .
$$

The last two relations imply that

$$
\xi \ln f=1 .
$$


More generally, we prove:

Theorem 5.1. If $M=M_{\perp} \times_{f} M_{T}$ is a warped product submanifold of a Kenmotsu manifold $\bar{M}$ with structure vector field tangential to $M_{\perp}$, then the warping function $f$ satisfies

$$
\nabla \ln f=\xi
$$

Proof. By (25), we have

$$
\left(\bar{\nabla}_{X} T\right) Z=A_{F Z} X+t h(X, Z)-\eta(Z) T X
$$

for each $X \in T M_{T}$ and $Z \in T M_{\perp}$. On using (51) in the left hand side of the above equation and taking product with $Y \in T M_{T}$ in both the sides, the equation reduces to

$$
(Z \ln f-\eta(Z)) g(X, T Y)=g(h(X, Y), F Z) .
$$

The expression in the left hand side of the above equation is skew symmetric in $X$ and $Y$ whereas the right hand side is symmetric $X$ and $Y$. Therefore,

$$
h(X, Y) \in \nu,
$$

and

$$
(Z \ln f-\eta(Z)) g(X, T Y)=0 .
$$

Now, as the submanifold is proper, we deduce from the last relation that

$$
Z \ln f=\eta(Z)
$$

for each $Z \in T M_{\perp}$. Now, as $\eta(C U)=0$ for each $U \in T M$, it follows from the above that $U \ln f=0$, for each $U \in T M-\langle\xi\rangle$, whereas by (53), $\xi \ln f=1$. This proves that

$$
\nabla \ln f=\xi .
$$

Hence assuming that $\xi=\frac{\partial}{\partial t}$, we infer from the above that on a warped product contact $C R$-submanifold of a Kenmotsu manifold, the warping function $f$ is given by $f(t)=e^{t}$.

Now, we may establish the following characterization.

Theorem 5.2. A contact CR-submanifold of a Kenmotsu manifold $\bar{M}$ is a warped product contact CR-submanifold if and only if there exists a smooth function $\mu$ on $M$ with $X \mu=0$ for each $X \in D$ satisfying the following formula

$$
\left(\bar{\nabla}_{U} T\right) V=g(T U, V) \nabla \mu-\eta(V) T U
$$

for each $U, V \in T M$

Proof. Let $M=M_{\perp} \times_{f} M_{T}$ be a warped product contact $C R$-submanifold of a Kenmotsu manifold $\bar{M}$. Then, for $X, Y \in T M_{T}$ and $Z \in T M_{\perp}$, by virtue of formula (11) and (49),

$$
g\left(\left(\bar{\nabla}_{X} T\right) Y, Z\right)=(Z \ln f) g(T X, Y),
$$


which on applying (16) takes the form

$$
\operatorname{nor}\left(\bar{\nabla}_{X} T\right) Y=g(T X, Y) \nabla \ln f,
$$

where 'nor' denotes the component of the underlying vector field tangential to $M_{\perp}$. On the other hand taking account of the fact that $t N \in T M_{\perp}$ for each $N \in T^{\perp} M$ in formula (25), we deduce that $B\left(\left(\bar{\nabla}_{X} T\right) Y\right)=0$. Equation (57) in view of this observation yields

$$
\left(\bar{\nabla}_{X} T\right) Y=g(T X, Y) \nabla \ln f .
$$

For arbitrary vector fields $U, V$ on $M$, by using (17) and (27), we may now, write

$\left(\bar{\nabla}_{U} T\right) V=\left(\bar{\nabla}_{B U} T\right) B V+\left(\bar{\nabla}_{B U} T\right) C V+\left(\bar{\nabla}_{C U} T\right) B V+\left(\bar{\nabla}_{C U} T\right) C V-\eta(V) T U$.

Substituting from (58), (50), (51), (52) and the fact that $C V \ln f=0$, we obtain

$$
\left(\bar{\nabla}_{U} T\right) V=g(T U, B V) \nabla \ln f-\eta(V) T U .
$$

This verifies the fact that on a warped product contact $C R$-submanifold of a Kenmotsu manifold equation (56) holds.

Conversely, suppose that $M$ is a contact $C R$-submanifold of a Kenmotsu manifold $\bar{M}$ such that for each $U, V \in T M$ and for a smooth function $\mu$ on $M$ satisfying $X \mu=0$ for all $X \in D$, formula (56) holds. Then for $Z, W \in D^{\perp} \oplus \xi$, it follows from (56)that

$$
\left(\bar{\nabla}_{Z} T\right) W=0
$$

Taking product with $X \in D$ in the above equation and using (11), we get

$$
g\left(\nabla_{Z} W, T X\right)=0 .
$$

This proves that $D^{\perp} \oplus\langle\xi\rangle$ is involutive and its leaves are totally geodesic in $M$.

Now, for $X, Y \in D$ and $Z \in D^{\perp} \oplus\langle\xi\rangle$, we have

$$
g(h(X, T Y), F Z)=-g(\operatorname{th}(X, T Y), Z) .
$$

Simplifying the right hand side by using (25), the above equation reduces to

$$
g(h(X, T Y), F Z)=-g\left(\left(\bar{\nabla}_{X} T\right) T Y, Z\right)+g(X, Y) \eta(Z) .
$$

On using (56) on the right hand side, the above equation takes the form:

$$
g(h(X, T Y), F Z)=(\eta(Z)-Z \mu) g(X, Y)=g(\xi-\nabla \mu, Z) g(X, Y),
$$

which shows that

$$
g(h(X, T Y), F Z)=g(h(T X, Y), F Z) .
$$

Hence, by Theorem 3.4, $D$ is involutive.

It is also easy to observe that $g\left(\nabla_{X} T Y, Z\right)=g\left(\left(\bar{\nabla}_{X} T\right) Y, Z\right)$ for each $X, Y \in$ $D$ and $Z \in D^{\perp} \oplus\langle\xi\rangle$, therefore on applying (56) we have,

$$
g\left(\nabla_{X} T Y, Z\right)=g(T X, Y) g(\nabla \mu, Z),
$$


which means

$$
\operatorname{nor}\left(\nabla_{X} T Y\right)=-g(X, T Y) \nabla \mu
$$

where, 'nor' denotes the component of the underlying vector field tangential to the leaves of $D^{\perp} \oplus\langle\xi\rangle$. In other words,

$$
h^{T}(X, Y)=-g(X, Y) \nabla \mu
$$

where $h^{T}$ denotes the second fundamental form of the immersion of $M_{T}$ into $M$. This means, that $M_{T}$ is totally umbilical in $M$ with $\nabla \mu$ as the mean curvature vector with respect to the immersion of $M_{T}$ into $M$. Further, as $X \mu=0$ for each $X \in D, \nabla \mu$ is parallel. That is, the leaves of $D$ are extrinsic spheres in $M$. Hence, by virtue of Theorem $4.2, M$ is locally a warped product $C R$-submanifold of $\bar{M}$. That is $M$ is a warped product $C R$-submanifold of $\bar{M}$.

A characterization in terms of the canonical structure $F$ is obtained in the following theorem:

Theorem 5.3. A contact CR-submanifold $M$ of a Kenmotsu manifold $\bar{M}$ is a warped product contact $C R$-submanifold if and only if there exist a $C^{\infty}$-function $\mu$ on $M($ with $X \mu=0$ for each $X \in D)$ such that

$$
\left(\bar{\nabla}_{U} F\right) V=f h(U, C V)-g(B U, B V) F \nabla \mu-\eta(V) F U
$$

for each $U, V \in T M$.

Proof. Let $M=M_{\perp} \times_{f} M_{T}$ be a warped product contact $C R$-submanifold of a Kenmotsu manifold $\bar{M}$. Then for $X, Y \in T M_{T}$ and $N \in T^{\perp} M$, on making use of(12), (49) and (16), we obtain that

$$
g\left(\left(\bar{\nabla}_{X} F\right) Y, N\right)=-g\left(F \nabla_{X} Y, N\right)=g(X, Y) g(F \nabla \ln f, N) .
$$

That gives

$$
\left(\bar{\nabla}_{X} F\right) Y=g(X, Y) F \nabla \ln f .
$$

Now, for $X \in T M_{T}$ and $Z \in T M_{\perp}$, formula (26) in view of the fact that $T Z=0=F X$ yields

$$
\left(\bar{\nabla}_{X} F\right) Z=f h(X, Z) .
$$

On the other hand by (49) and (12), we have

$$
\left(\bar{\nabla}_{Z} F\right) X=0 \text {. }
$$

Now for $U, V \in T M$, we may write

$\left(\bar{\nabla}_{U} F\right) V=\left(\bar{\nabla}_{B U} F\right) B V+\left(\bar{\nabla}_{B U} F\right) C V+\left(\bar{\nabla}_{C U} F\right) B V+\left(\bar{\nabla}_{C U} F\right) C V-\eta(V) F U$.

Substituting the values of the terms appearing in the right hand side of the above equation from $(63),(64),(65)$ and $(26)$ we obtain

$$
\left(\bar{\nabla}_{U} F\right) V=-g(B U, B V) F \nabla \ln f+f h(U, C V)-\eta(V) F U .
$$

This verifies (62). 
Conversely suppose that $M$ is a contact $C R$-submanifold of a Kenmotsu manifold such that formula (62) holds with $X \mu=0$ for each $X \in D$, then

$$
\left(\bar{\nabla}_{X} F\right) Y=-g(X, Y) F \nabla \mu
$$

and by (26),

$$
h(X, T Y)=f h(X, Y)-\left(\bar{\nabla}_{X} F\right) Y
$$

for each $X, Y \in D$. Since the right hand side of the above equation is symmetric in $X$ and $Y$ (due to (66) and the symmetry of $h$ ), $h(X, T Y)=h(T X, Y)$. Therefore, by Theorem 3.4, $D$ is involutive on $M$. Further, it follows from equation (66) and (12) that

$$
C\left(\nabla_{X} Y\right)=-g(X, Y) C(\nabla \mu) .
$$

If $M_{T}$ is a leaf of $D$ and $h^{T}$ is the second fundamental form of $M_{T}$ into $M$, then from the last equation we have

$$
h^{T}(X, Y)=g(X, Y) \nabla \mu .
$$

Hence, we conclude that the leaves of $D$ are totally umbilical in $M$ with mean curvature vector $\nabla \mu$. Further as $X \mu=0$, each leaf of $D$ is an extrinsic sphere in $M$.

Now for $Z, W \in D^{\perp} \oplus\langle\xi\rangle$ and $X \in D$

$$
g\left(\nabla_{Z} W, X\right)=-g\left(F \nabla_{Z} X, F W\right)=g\left(\left(\bar{\nabla}_{Z} F\right) X, F W\right) .
$$

The right hand side of the above equation is zero by virtue of (62). That proves

$$
g\left(\nabla_{Z} W, X\right)=0,
$$

which means $D^{\perp} \oplus\langle\xi\rangle$ is parallel. In other words, $D^{\perp} \oplus\langle\xi\rangle$ is involutive and its leaves are totally geodesic in $M$. Hence, by virtue of Theorem $4.2, M$ is locally a warped product $C R$-submanifold of $\bar{M}$. That is $M=M_{\perp} \times_{\mu} M_{T}$ is warped product $C R$-submanifold of $\bar{M}$.

Example $5.4([12])$. Consider the complex space $\mathbb{C}^{4}$ with the usual Kaehler structure and the real global coordinates $\left(x^{1}, y^{1}, \ldots, x^{4}, y^{4}\right)$. Let $\bar{M}=\mathbb{R} \times{ }_{f} \mathbb{C}^{4}$ with warping function $f=e^{t}, t$ being the global coordinate on $\mathbb{R}$. Then $\bar{M}$ is Kenmotsu manifold.

Consider the distributions

$$
D=\operatorname{span}\left\{\frac{\partial}{\partial x^{1}}, \frac{\partial}{\partial y^{1}}, \frac{\partial}{\partial x^{2}}, \frac{\partial}{\partial y^{2}}\right\}, D^{\perp}=\operatorname{span}\left\{\frac{\partial}{\partial t}, \frac{\partial}{\partial x^{3}}, \frac{\partial}{\partial x^{4}}\right\} .
$$

Let $N_{T}$ and $N_{\perp}$ be the leaves of $D$ and $D^{\perp}$, respectively, and

$$
g_{N_{T}}=\sum_{i=1}^{2}\left(\left(d x^{i}\right)^{2}+\left(d y^{i}\right)^{2}\right)
$$

and

$$
g_{N_{\perp}}=d t^{2}+e^{2 t} \sum_{\alpha=3}^{4}\left(d x^{\alpha}\right)^{2}
$$


be the Riemannian metrics on $N_{T}$ and $N_{\perp}$, respectively. Then $M=N_{\perp} \times_{f} N_{T}$ is a warped contact $C R$-submanifold of $\bar{M}$. The warping function is given by $f=e^{t}$. The above example can also be generalized on replacing $\mathbb{C}^{4}$ by $\mathbb{C}^{n}$ and modifying the distributions $D$ and $D^{\perp}$, respectively.

\section{References}

[1] R. L. Bishop and B. O'Neil, Manifolds of negative curvature, Trans. Amer. Math. Soc. $145(1969), 1-9$.

[2] B. Y. Chen, CR-submanifold of Kaehler manifolds. I, J. Differential Geom. 16 (1981), no. $2,305-323$.

[3] _ Slant Immersion, Bull. Autral. Math. Soc. 41 (1990), no. 1, 135-147.

[4] - Geometry of warped product CR-submanifolds in Kaehler manifolds, Montash. Math. 133 (2001), no. 3, 177-195.

[5] I. Hasegawa, I. Mihai, Contact CR-warped product submanifolds in Sasakian manifolds, Geom. Dedicata 102 (2003), 143-150.

[6] S. Hiepko, Eine innere Kennzeichnung der verzerrten Produkte, Math. Ann. 241 (1979), no. 3, 209-215.

[7] S. T. Hong, Warped product and black holes, Nuovo Cimento Soc. Ital. Fis. B 120 (2005), no. 10-11, 1221-1234.

[8] K. Kenmotsu, A class of almost contact Riemannian manifolds, Tohoku Math. 24 (1972), 93-103.

[9] V. A. Khan, K. A. Khan, and Siraj-Uddin, CR-Warped product submanifolds in a Kaehler Manifold, Southeast Asian Bull. Math. 33 (2009), no. 5, 865-874.

[10] M. Kobayashi, Semi-invariant submanifolds of a certain class of almost contact manifolds, Tensor (N.S.) 43 (1986), no. 1, 28-36.

[11] M. I. Munteanu, Warped product contact CR-submanifold of Sasakian space form, Publ. Math. Debreen 66 (2005), no. 1-2, 75-120.

[12] _ A note on doubly warped product contact CR-submanifold in trans-Sasakian manifolds, Acta Math. Hungar. 16 (2007), no. 1-2, 121-126.

[13] J. A. Oubina, New class of almost contact metric structures, Publ. Math. Debreen 32 (1985), no. 3-4, 187-193.

[14] S. Tanno, The automorphism groups of almost contact Riemannian manfolds, Tohoku Math. J. 21 (1969), 21-38.

[15] K. Yano and M. Kon, CR-Submanifolds of Kaehlerian and Sasakian Manifolds, Birkhauser Verlag, Boston, 1983.

ViqAR AZAM Khan

Department of Mathematics

Aligarh Muslim University

Algarh 202002, India

E-mail address: viqarster@gmail.com

Mohammad Shuaib

Department of Mathematics

Aligarh Muslim University

Algarh 202002, India

E-mail address: shuaibyousuf6@gmail.com 